\title{
Anabases
}

ANABASES Traditions et réceptions de l'Antiquité

$23 \mid 2016$

Varia

\section{Jonathan ZARECKI, Cicero's Ideal Statesman in Theory and Practice}

\section{Amedeo Alessandro Raschieri}

\section{OpenEdition}

\section{Journals}

Edizione digitale

URL: http://journals.openedition.org/anabases/5717

DOI: 10.4000/anabases.5717

ISSN: 2256-9421

\section{Editore}

E.R.A.S.M.E.

\section{Edizione cartacea}

Data di pubblicazione: 2 maggio 2016

Paginazione: 337-339

ISSN: 1774-4296

Notizia bibliografica digitale

Amedeo Alessandro Raschieri, « Jonathan zaREckI, Cicero's Ideal Statesman in Theory and Practice », Anabases [Online], 23 | 2016, Messo online il 02 mai 2016, consultato il 22 septembre 2020. URL : http://journals.openedition.org/anabases/5717 ; DOI : https://doi.org/10.4000/anabases.5717

Questo documento è stato generato automaticamente il 22 settembre 2020.

(c) Anabases 


\title{
Jonathan ZARECKI, Cicero's Ideal Statesman in Theory and Practice
}

\author{
Amedeo Alessandro Raschieri
}

\section{NOTIZIA}

Jonathan ZARECKI, Cicero's Ideal Statesman in Theory and Practice

London, Bloomsbury, 2014, $212 \mathrm{p}$.

60 livres/ ISBN 978-1-78093-295-8

1 Il volume di Zarecki presenta, in un dettagliato affresco narrativo, la biografia politica di Cicerone intrecciata alla sua vita privata e alla sua attività intellettuale. Al centro dell'analisi è posto il problema del "rector-ideal ", cioè, come è evidente dal titolo stesso, la costruzione teorica del governante ideale e il confronto con le sue varie personificazioni a Roma durante l'età tardo-repubblicana. Nonostante un prevalente interesse storico e letterario, la trattazione riesce a coinvolgere in modo efficace il contesto filosofico (in particolare l'adesione ciceroniana allo scetticismo accademico) e a rintracciare la valenza pragmatica di tale figura. Oltre al fatto di prendere una posizione chiara e personale nei confronti delle interpretazioni moderne sulla questione (quelle di R. Reitzenstein, E. Meyer, R. Heinze, E. Lepore, P. Krarup e, in tempi più recenti, quelle di A. Lintott, K. Girardet, T. Stevenson, W. Stroh, J.G.F. Powell, J.E.G. Zetzel e E. Fantham), il merito principale di Zarecki è l'ampliamento prospettico dall'immagine del rector nel De re publica al complesso dell'attività politica e della produzione letteraria ciceroniane.

2 Dopo l'introduzione, dedicata a chiarire gli aspetti generali e metodologici, la trattazione è suddivisa in cinque capitoli, organizzati perlopiù in modo diacronico. Nel primo è affrontato il tema dell'educazione filosofica di Cicerone con la sua adesione allo scetticismo che, nel caso del De re publica, consente, nel momento del fallimento del tradizionale ordinamento costituzionale, di svolgere un'apologia della monarchia e di delineare la figura del rector attraverso un dialogo di tipo accademico. Nel secondo 
capitolo è analizzato il contesto sociale, culturale e politico che ha permesso la composizione del De re publica e, in particolare, sono distinti tre momenti : la dissoluzione della concordia ordinum nel periodo tra il consolato di Cicerone e il suo esilio, gli anni tra il ritorno dall'esilio e l'inizio della stesura del De re publica (con l'analisi approfondita del De oratore) e il periodo tra la pubblicazione del De oratore e la partenza di Cicerone per il proconsolato in Cilicia (con una particolare attenzione per l'emergere della figura di Pompeo e per la sua influenza sulla riflessione ciceroniana contenuta nel De re publica e nel De legibus).

Il terzo capitolo è dedicato alla figura ideale del rector come costruzione teorica e pratica, come antitesi del tiranno e come vir bonus dotato di sapientia, prudentia e auctoritas. Per Cicerone un singolo rector, come figura autocratica, è al lavoro nello Stato in ogni periodo della storia romana e, alla luce di tale immagine ideale, è analizzata la posizione ciceroniana nel corso della guerra civile tra Cesare e Pompeo. Il quarto capitolo è incentrato sul confronto con l'autocrazia di Cesare : durante l'esilio volontario a Brindisi, nel Cato e nelle orazioni Pro Marcello e Pro Ligario, Cicerone utilizza l'ideale del rector in funzione di opposizione e critica verso il dittatore. L'ultimo capitolo riguarda, invece, le opere filosofiche del 44 e la lotta contro Antonio : nel De senectute e nel De amicitia, ma soprattutto nel De officiis e nelle Filippiche, la figura di Antonio diventa, in modo più o meno esplicito, l'antitesi del rector e, di conseguenza, Cicerone stesso cerca di incarnare il suo ideale politico, a costo di un fallimento completo e della perdita della propria vita. In chiusura, un breve epilogo sottolinea gli aspetti principali e innovativi della trattazione, ben riassunti in queste parole : « my evaluation of the rector-ideal as a practical code of behavior for Cicero and the paradigm by which he assessed the actions of his contemporaries provides a new interpretation of this enigmatic concept » (162).

4 Il volume è contraddistinto da una struttura argomentativa chiara ed efficace, anche se talvolta, con i frequenti schemi riassuntivi e i rimandi interni, risente di uno stile da dissertazione (in effetti, il lavoro ha preso l'avvio dalle ricerche dottorali di Zarecki presso l'University of Florida, continuate poi presso altre istituzioni statunitensi). Di particolare interesse è l'attenzione mostrata per il lessico latino nei suoi aspetti storicolinguistici e semantici e per il problema della traduzione in inglese (per esempio, quella dei termini rector, res publica e clementia). Ampio spazio è poi riservato all'analisi, anche puntuale e approfondita, sugli aspetti contenutistici e stilistici dei brani ciceroniani utilizzati come fonti. Il lavoro si basa, inoltre, su un esteso impiego di materiale tratto dall'epistolario ciceroniano, fondamentale per seguire l'evoluzione del pensiero e della pratica politica dell'autore antico. Efficace e originale è pure la selezione delle opere filosofiche, retoriche e oratorie - impiegate per confermare ed estendere l'indagine sulle componenti più specificatamente politiche del pensiero ciceroniano.

Qualche riserva rimane sulla scelta editoriale di raccogliere le note alla fine del volume (con una formattazione peraltro approssimativa), cosicché risultano poco agevoli il controllo dei riferimenti bibliografici e gli ulteriori approfondimenti che il libro stimola nel lettore. A tal fine sono, invece, molto utili l'accurato Index locorum, che tra l'altro mostra l'ampiezza e la validità delle fonti primarie, e il General index, che costituisce un'agile guida attraverso i capisaldi del pensiero politico ciceroniano. La bibliografia, aggiornata al 2013, nonostante una comprensibile preferenza per gli studi di area anglosassone, comprende anche i più significativi lavori in francese, tedesco e italiano. In definitiva, la lettura del volume è raccomandata non solo agli specialisti di Cicerone, 
ma anche agli studiosi del pensiero politico, antico e moderno, e a un pubblico più ampio interessato al mondo romano nei suoi aspetti storici e culturali.

\section{AUTORE}

\section{AMEDEO ALESSANDRO RASCHIERI}

Università degli Studi di Milano

amedeo.raschieri@alice.it 